\title{
Further extensions to rectangular dielectric waveguide technique for dielectric
} measurements

\begin{abstract}
This paper describes a method to obtain the dielectric constant of materials using the rectangular dielectric waveguide technique in the WR-22 frequency band. By applying the solution of the wave equation, the actual dielectric constant for samples of small transverse dimensions can be directly recovered from the measured effective dielectric constant by a numerical technique. The method is very quick and simple and provides sufficient accuracy for most practical purposes
\end{abstract}

